Publ. Mat. 60 (2016), 311-334

DOI: 10.5565 /PUBLMAT_60216_03

\title{
VITALI'S THEOREM WITHOUT UNIFORM BOUNDEDNESS
}

\author{
Nguyen Quang Dieu, Phung Van Manh, Pham Hien Bang, \\ AND LE THANH Hung
}

This work is dedicated to the 75th birthday of Professor Nguyen Van Khue

\begin{abstract}
Let $\left\{f_{m}\right\}_{m>1}$ be a sequence of holomorphic functions defined on a bounded domain $D \subset \mathbb{C}^{n}$ or a sequence of rational functions $\left(1 \leq \operatorname{deg} r_{m} \leq m\right)$ defined on $\mathbb{C}^{n}$. We are interested in finding sufficient conditions to ensure the convergence of $\left\{f_{m}\right\}_{m>1}$ on a large set provided the convergence holds pointwise on a not too small set. This type of result is inspired from a theorem of Vitali which gives a positive answer for uniformly bounded sequence.
\end{abstract}

2010 Mathematics Subject Classification: Primary: 41A05, 41A63, 46A32.

Key words: Rapid convergence, convergence in capacity, pluripolar set, relative capacity.

\section{Introduction}

Let $D$ be a domain in $\mathbb{C}^{n},\left\{f_{m}\right\}_{m \geq 1}$ be a sequence of holomorphic functions defined on $D$. A classical theorem of Vitali asserts that if $\left\{f_{m}\right\}_{m \geq 1}$ is uniformly bounded on compact subsets of $D$ and if the sequence is pointwise convergent to a function $f$ on a subset $X$ of $D$ which is not contained in any complex hypersurface of $D$, then $\left\{f_{m}\right\}_{m \geq 1}$ converges uniformly on compact subsets of $D$. A striking feature of this theorem is that we may extend, in some sense, the function $f$ which is a priori measurable and defined on a very small set, to a holomorphic function entirely on $D$. We note, however, that the assumption on uniform boundedness of $\left\{f_{m}\right\}_{m \geq 1}$ is essential. Indeed, using the classical Runge approximation theorem, it is possible to construct a sequence of polynomials on $\mathbb{C}$ that converges pointwise to 0 everywhere except at the origin where the limit is 1 ! In particular, the convergence is not uniform on any open set containing the origin. For details, see Example 1 in [6]. We are concerned with finding analogues of the mentioned above 
theorem of Vitali in which the uniform boundedness of the sequence under consideration is omitted. A possible approach is to impose stronger mode of convergence and/or the size of $X$. Motivated by the problem of finding local conditions for single-valuedness of holomorphic continuation, Gonchar proved in Theorem 2 of [7] the following remarkable result.

Theorem 1.1. Let $\left\{r_{m}\right\}_{m \geq 1}$ be a sequence of rational functions in $\mathbb{C}^{n}$ $\left(\operatorname{deg} r_{m} \leq m\right)$ that converges rapidly in measure on an open set $X$ to a holomorphic function $f$ defined on a bounded domain $D(X \subset D)$ i.e., for every $\varepsilon>0$

$$
\lim _{m \rightarrow \infty} \lambda_{2 n}\left(z \in X:\left|r_{m}(z)-f(z)\right|^{1 / m}>\varepsilon\right)=0 .
$$

Here $\lambda_{2 n}$ is the Lebesgue measure in $\mathbb{C}^{n} \cong \mathbb{R}^{2 n}$. Then $\left\{r_{m}\right\}_{m \geq 1}$ must converge rapidly in measure to $f$ on the whole domain $D$.

Much later, by using techniques of pluripotential theory, Bloom was able to prove an analogous result in which rapidly convergence in measure is replaced by rapidly convergence in capacity and the set $X$ is only required to be compact and non-pluripolar (see Theorem 2.1 in $[3]$ ). More precisely, we have

Theorem 1.2. Let $f$ be a holomorphic function defined on a bounded domain $D \subset \mathbb{C}^{n}$. Let $\left\{r_{m}\right\}_{m \geq}$ be a sequence of rational functions $\left(\operatorname{deg} r_{m} \leq m\right)$ converging rapidly in capacity to $f$ on a non-pluripolar Borel subset $X$ of $D$ i.e., for every $\varepsilon>0$

$$
\lim _{m \rightarrow \infty} \operatorname{cap}\left(\left\{z \in X:\left|r_{m}(z)-f(z)\right|^{1 / m}>\varepsilon\right\}, D\right)=0 .
$$

Then $\left\{r_{m}\right\}_{m \geq 1}$ converges to $f$ rapidly in capacity on $D$ i.e., for every Borel subset $E$ of $D$ and for every $\varepsilon>0$

$$
\lim _{m \rightarrow \infty} \operatorname{cap}\left(\left\{z \in E:\left|r_{m}(z)-f(z)\right|^{1 / m}>\varepsilon\right\}, D\right)=0 .
$$

Here $\operatorname{cap}(., D)$ denotes the relative capacity; a brief discussion of this kind of capacity as well as convergence in capacity will be given in the next section. Using a standard result which relates convergence in capacity and pointwise convergence (cf. Lemma 2.2), it is not hard to check that Theorem 1.1 follows from Theorem 1.2 (see Theorem 2.2 in [3]). The above theorems of Gonchar and Bloom are the main inspiration for our research. The first result of this paper, Theorem 3.1, states roughly that if a sequence of bounded holomorphic functions is convergence fast enough on a non-pluripolar set then the convergence is also fast uniformly on compact sets. Here the speed of approximation is measured 
in terms of the growth of the sup-norms of $f_{m}$. The next result, much in the same spirit of Theorems 1.1 and 1.2, deals with a version of Vitali's theorem for a sequence of rational functions. In Theorem 3.4 we consider a sequence $\left\{r_{m}\right\}_{m \geq 1}$ of rational functions that is rapidly pointwise convergent on a Borel non-pluripolar subset of $\mathbb{C}^{n}$ to a bounded measurable function. Under an additional condition that the degree of the denominator of $r_{m}$ tends to $\infty$ much less than $m$, we are able to show that the sequence $\left\{r_{m}\right\}_{m \geq 1}$ converges rapidly in measure entirely on $\mathbb{C}^{n}$ to a measurable function $F$. The main result of the paper (Theorem 3.6), to some extent, is a generalization of Theorem 1.1 and Theorem 1.2 when the sequence $\left\{r_{m}\right\}_{m \geq 1}$ is supposed to converge rapidly to radial boundary values of some bounded holomorphic function $f$ defined on a bounded domain $D \subset \mathbb{C}^{n}$. More precisely, we show that if the subset where the convergence occurs is not too small then the same type of convergence must hold inside the domain. Moreover, we also consider the convergence of $\left\{r_{m}\right\}_{m \geq 1}$ to $f$ on affine subspaces of $\mathbb{C}^{n}$. As an illustration of this theorem, we establish in Proposition 4.2 an example of a bounded holomorphic function $f$ on the unit disk $\Delta$ and a sequence of rational functions $\left\{r_{m}\right\}_{m \geq 1}$ with poles lying outside $\bar{\Delta}$ such that $\left\{r_{m}\right\}_{m \geq 1}$ converges rapidly pointwise to $f^{*}$, the radial boundary values of $f$, on a compact subset $F \subset \partial \Delta$ of positive length. Nevertheless, the function $f$ does not extend holomorphically through any point of $F$.

Acknowledgements. This work has been started during a visit of the first named author at the Vietnam Institute for Advanced Mathematics (VIASM) in the winter of 2012. The paper was finally completed during a stay of the first and the second named authors at VIASM in the winter of 2014. We wish to thank VIASM for financial support and the warm hospitality. It is also our pleasure to thank Professor Pascal Thomas for a useful suggestion in the construction of Proposition 4.2. Last but not least, we are grateful to an anonymous referee for her/his constructive comments, especially for asking a clever question that leads to the current state of our main result (Theorem 3.6). The first named author is supported by the grant 101.02-2013.11 from the NAFOSTED program. The second named author is also sponsored by the NAFOSTED program under the grant number 101.02-2014.01.

\section{Preliminaries}

For the reader convenience, we collect in this preparatory section necessary elements of pluripotential theory that will be needed later on. Let $D$ be a domain in $\mathbb{C}^{n}$. An upper semicontinuous function 
$u: D \rightarrow[-\infty, \infty)$ is said to be plurisubharmonic if the restriction of $u$ on $D \cap l$ is subharmonic for every complex line $l$. The cone of plurisubharmonic function on $D$ is denoted by $\operatorname{PSH}(D)$. A subset $E$ of $\mathbb{C}^{n}$ is said to be pluripolar if for every $z_{0} \in E$ there exists an open connected neighborhood $U$ of $z_{0}$ and $u \in \operatorname{PSH}(U), u \neq \equiv-\infty$ such that $u \equiv-\infty$ on $E \cap U$. According to a classical theorem of Josefson, if $E$ is pluripolar then there exists a plurisubharmonic function $u$ which defined globally on $\mathbb{C}^{n}$ such that $u \equiv-\infty$ on $E$. Clearly a proper complex subvariety of $D$ is pluripolar. On the other hand, it is not hard to show that any subset of $\mathbb{C}^{n}$ with positive Lebesgue measure is not pluripolar.

In order to measure how close a Borel set to be pluripolar, following Bedford and Taylor (see $[\mathbf{9}$, p. 120]) we let $\operatorname{cap}(E, D)$ be the relative capacity of a Borel subset $E$ in $D$ which is defined as

$$
\operatorname{cap}(E, D)=\sup \left\{\int_{E}\left(d d^{c} u\right)^{n}: u \in \operatorname{PSH}(D),-1<u<0\right\} .
$$

It is well known that relative capacity enjoys some important properties such as sub-additivity and monotone under increasing sequences. Moreover, a deep result in Bedford-Taylor's theory states that pluripolar subsets of $D$ are exactly subsets with vanishing relative capacity. We also frequently appeal to Bernstein-Walsh's inequality (see [11]) which states that if $K, L$ are compact sets in $\mathbb{C}^{n}$ and $K$ is non-pluripolar, then there exists $C_{K, L}>0$ depending only on $K$ and $L$ such that for any polynomial $p_{m}$ in $\mathbb{C}^{n}$ of degree at most $m$,

$$
\frac{1}{m} \log \left\|p_{m}\right\|_{L} \leq \frac{1}{m} \log \left\|p_{m}\right\|_{K}+C_{K, L} .
$$

We recall the following types of convergence of measurable functions which are essentially well known.

Definition 2.1. Let $\left\{f_{m}\right\}_{m \geq 1}, f$ be complex valued, measurable functions defined on a bounded domain $D \subset \mathbb{C}^{n}$. We say that the sequence $\left\{f_{m}\right\}_{m \geq 1}$

(i) converges in capacity to $f$ on $X$ if for every $\varepsilon>0$ we have

$$
\lim _{m \rightarrow \infty} \operatorname{cap}\left(X_{m, \varepsilon}, D\right)=0,
$$

where $X_{m, \varepsilon}:=\left\{x \in X:\left|f_{m}(x)-f(x)\right|>\varepsilon\right\}$

(ii) converges in capacity to $f$ on $D$ if property (i) holds true for every compact subset $X$ of $D$.

We have the following relation between convergence in capacity and pointwise convergence. 
Lemma 2.2. Let $\left\{f_{m}\right\}_{m \geq 1}$ and $f$ be complex valued, measurable functions defined on a domain $D \subset \mathbb{C}^{n}$. If $\left\{f_{m}\right\}_{m \geq 1}$ converges in capacity to $f$ on a Borel subset $X$ of $D$, then there exists a subsequence $\left\{f_{m_{j}}\right\}_{j \geq 1}$ and a pluripolar subset $E \subset X$ such that $\left\{f_{m_{j}}\right\}_{j \geq 1}$ converges pointwise to $f$ on $X \backslash E$.

Proof: Suppose that $\left\{f_{m}\right\}_{m \geq 1}$ is a sequence that converges in capacity to $f$ on $X$. For every $\varepsilon>0$, the hypothesis gives

$$
\lim _{m \rightarrow \infty} \operatorname{cap}\left(X_{m, \varepsilon}, D\right)=0,
$$

where $X_{m, \varepsilon}:=\left\{x \in X:\left|f_{m}(x)-f(x)\right|>\varepsilon\right\}$. Hence, we can find a strictly increasing sequence $\left\{m_{k}\right\}$ such that

$$
\operatorname{cap}\left(X_{m, \frac{1}{2^{k}}}, D\right)<\frac{1}{2^{k}}, \quad \forall m \geq m_{k} .
$$

Let us set $E_{j}:=\bigcup_{k=j}^{\infty} X_{m_{k}, \frac{1}{2^{k}}}$ and $E:=\bigcap_{j=1}^{\infty} E_{j}$. By the sub-additive property of the relative capacity we obtain

$\operatorname{cap}(E, D) \leq \operatorname{cap}\left(E_{j}, D\right) \leq \sum_{k=j}^{\infty} \operatorname{cap}\left(X_{m_{k}, \frac{1}{2^{k}}}, D\right)<\sum_{k=j}^{\infty} \frac{1}{2^{k}}=\frac{1}{2^{j-1}}, \quad \forall j \geq 1$.

It follows that $\operatorname{cap}(E, D)=0$ and hence $E$ is a pluripolar set. Now, for $z \in X \backslash E$, it is easy to check using the definition of $E_{j}$ that $\lim _{k \rightarrow \infty} f_{m_{k}}(z)=f(z)$. We are done.

We should mention that there exists a pointwise convergence sequence that contains no subsequence that converges in capacity. Indeed, let $\left\{A_{m}\right\}_{m \geq 1}$ be a sequence of pairwise disjoint subsets of the unit disk $\Delta \subset$ $\mathbb{C}$ such that $\inf _{m \geq 1} \operatorname{cap}\left(A_{m}, \Delta\right)>0$. Then the sequence of characteristic functions $\left\{\chi_{A_{m}}\right\}_{m \geq 1}$ provides the desired example. It should be remarked that we do not know if there exists a version of Egorov's theorem for convergence in capacity, i.e. every pointwise convergence sequence contains a subsequence that converges in capacity on some non-pluripolar subset.

We will frequently appeal to the following fundamental result of Bedford and Taylor (Theorem 4.7.6 in [9]) which partly explains the role of pluripolar sets in pluripotential theory.

Lemma 2.3. Let $\left\{u_{m}\right\}_{m \geq 1}$ be a sequence of plurisubharmonic functions on $D$. Assume that the sequence is uniformly bounded from above on compact sets of D. Let

$$
u(z):=\limsup _{m \rightarrow \infty} u_{m}(z), \quad z \in D .
$$

Then the set $\left\{z \in D: u(z)<u^{*}(z)\right\}$ is pluripolar. 


\section{Rapid convergence of holomorphic functions and rational functions}

We start with the following generalization of Vitali's theorem mentioned in the introduction.

Theorem 3.1. Let $D$ be a domain in $\mathbb{C}^{n}$ and $\left\{f_{m}\right\}_{m \geq 1}$ be a sequence of bounded holomorphic functions on D. Suppose that there exists an increasing sequence $\left\{\alpha_{m}\right\}_{m \geq 1}$ of positive numbers satisfying the following properties:

(i) $\left\|f_{m+1}-f_{m}\right\|_{D} \leq e^{\alpha_{m}}$.

(ii) $\alpha:=\inf _{m \geq 1}\left(\alpha_{m+1}-\alpha_{m}\right)>0$.

(iii) There exists a non-pluripolar Borel subset $X$ of $D$ and a bounded measurable function $f: X \rightarrow \mathbb{C}$ such that

$$
\left|f_{m}(x)-f(x)\right|^{1 / \alpha_{m}} \rightarrow 0, \quad \forall x \in X .
$$

Then the following assertions hold:

(a) $\left\{f_{m}\right\}_{m \geq 1}$ converges uniformly on compact sets of $D$ to a holomorphic function $f$.

(b) For every compact subset $K$ of $D$ we have $\lim _{m \rightarrow \infty}\left\|f_{m}-f\right\|_{K}^{1 / \alpha_{m}}=0$.

Proof: We define for $m \geq 1$ the function

$$
u_{m}(z):=\frac{1}{\alpha_{m}} \log \left|f_{m+1}(z)-f_{m}(z)\right|, \quad \forall z \in D .
$$

It follows from assumption (i) that $\left\{u_{m}\right\}_{m \geq 1}$ is a sequence of plurisubharmonic functions on $D$ which is uniformly bounded from above on $D$. We claim that

$$
\lim _{m \rightarrow \infty} u_{m}(x)=-\infty, \quad \forall x \in X .
$$

For this, fix $x \in X$ and $\varepsilon \in(0,1)$. By (3.1), there exists $m_{0} \geq 1$ such that

$$
\left|f_{m}(x)-f(x)\right|<\varepsilon^{\alpha_{m}}, \quad m \geq m_{0} .
$$

This implies that

$$
u_{m}(x) \leq \frac{1}{\alpha_{m}} \log \left(\varepsilon^{\alpha_{m}}+\varepsilon^{\alpha_{m+1}}\right)<\frac{\log 2}{m}+\log \varepsilon, \quad \forall m \geq m_{0} .
$$

The claim follows by letting $\varepsilon \downarrow 0$. Now we set

$$
u:=\left(\limsup _{m \rightarrow \infty} u_{m}\right)^{*} .
$$

Then $u \in \operatorname{PSH}(D)$. Furthermore, by the claim proven above and Lemma 2.3 we infer that $u=-\infty$ on a non-pluripolar subset of $X$. Thus $u \equiv-\infty$ on $D$. In particular $u_{m}$ converges pointwise to $-\infty$ on $D$. 
Next, by Hartogs' lemma we conclude that $u_{m}$ tends uniformly to $-\infty$ on compact sets of $D$. It follows that, given a compact subset $K$ of $D$, for every constant $A>0$, there exists $m(A) \geq 1$ such that

$$
\left\|f_{m+1}-f_{m}\right\|_{K}<e^{-A \alpha_{m}}, \quad \forall m \geq m(A) .
$$

Using (ii) and the triangle inequality we obtain

$\left\|f_{m+n}-f_{m}\right\|_{K}<\sum_{0 \leq k \leq n-1} e^{-A \alpha_{m+k}}<e^{-A \alpha_{m}}\left(\sum_{k \geq 0} e^{-A k \alpha}\right)=\frac{e^{-A \alpha_{m}}}{1-e^{-A \alpha}}$

Therefore, we may apply Cauchy's criterion to deduce that $\left\{f_{m}\right\}_{m \geq 1}$ converges uniformly on compact sets of $D$ to a holomorphic function $f$. Moreover, by letting $n \rightarrow \infty$ in the above inequality and observing that $A$ can be made arbitrarily large, we get the desired rapid uniform convergence on $K$. The proof is complete.

Corollary 3.2. Let $\left\{p_{m}\right\}_{m \geq 1}$ be a sequence of polynomials in $\mathbb{C}^{n}$ with $\operatorname{deg} p_{m} \leq m$. Assume that there exists a non-pluripolar Borel subset $X$ of $\mathbb{C}^{n}$ and a measurable function $f: X \rightarrow \mathbb{C}$ such that

$$
\left|p_{m}(x)-f(x)\right|^{1 / m} \rightarrow 0, \quad \forall x \in X .
$$

Then the following assertions hold:

(a) $\left\{p_{m}\right\}_{m \geq 1}$ converges uniformly on compact sets of $\mathbb{C}^{n}$ to a holomorphic function $f$.

(b) For every compact subset $K$ of $\mathbb{C}^{n}$ we have $\lim _{m \rightarrow \infty}\left\|p_{m}-f\right\|_{K}^{1 / m}=0$.

Proof: Let $D$ be a relatively compact domain in $\mathbb{C}^{n}$. According to Theorem 3.1, it suffices to prove that on the domain $D$, the sequence $\left\{p_{m}\right\}_{m \geq 1}$ satisfies the conditions given in Theorem 3.1. For this, we set

$$
X_{N}=\left\{z \in X:\left|p_{m}(z)-f(z)\right|^{1 / m} \leq N, \forall m \geq 1\right\}, \quad N \geq 1 .
$$

By (3.2), we have $\bigcup_{N>1} X_{N}=X$. Since $X$ is non-pluripolar, we infer that there must exist $\bar{N}_{0}$ such that $X_{N_{0}}$ is non-pluripolar. Since $X_{N_{0}}$ is Borel, it must contain a non-pluripolar compact subset $X^{\prime}$. Since $f$ is bounded on $X$, we obtain

$$
\sup _{m \geq 1} \frac{1}{m} \log \left\|p_{m}\right\|_{X^{\prime}}<\infty .
$$

Thus, using Bernstein-Markov's inequality we get

$$
\sup _{m \geq 1} \frac{1}{m} \log \left\|p_{m}\right\|_{D}<\infty \text {. }
$$


By simple estimates we get

$$
\left\|p_{m+1}-p_{m}\right\|_{D} \leq e^{C m}, \quad \forall m \geq 1,
$$

where $C>0$ is a constant independent of $m$. Thus the sequence $\alpha_{m}:=$ $\mathrm{Cm}$ satisfies conditions given in Theorem 3.1. We are done.

The situation is technically complicated for sequences of rational functions because of the presence of poles sets. First, we need the following concept.

Definition 3.3. Let $V$ be an algebraic hypersurface in $\mathbb{C}^{n}$ and $U$ be an open subset of $\mathbb{C}^{n}$. We define the degree of $V \cap U$ to be least integer $d$ so that there exists a polynomial $p$ of degree $d$ in $\mathbb{C}^{n}$ such that $V \cap U=$ $\{z \in U: p(z)=0\}$.

Now we are able to formulate our next result.

Theorem 3.4. Let $\left\{r_{m}\right\}_{m \geq 1}$ be a sequence of rational functions on $\mathbb{C}^{n}$ satisfying the following properties:

(i) There exist a Borel non-pluripolar subset $X$ of $\mathbb{C}^{n}$ and a bounded measurable function $f: X \rightarrow \mathbb{C}$ such that

$$
\lim _{m \rightarrow \infty}\left|r_{m}(x)-f(x)\right|^{1 / m}=0, \quad \forall x \in X .
$$

(ii) For every $z_{0} \in \mathbb{C}^{n}$, there exist an open ball $\mathbb{B}\left(z_{0}, r\right), m_{0} \geq 1$, and $\lambda \in(0,1)$ such that

$$
\operatorname{deg}\left(V_{m} \cap \mathbb{B}\left(z_{0}, r\right)\right) \leq m^{\lambda}, \quad \forall m \geq m_{0},
$$

where $V_{m}$ denotes the pole sets of $r_{m}$.

Then there exists a measurable function $F: \mathbb{C}^{n} \rightarrow \mathbb{C}$ such that $\mid r_{m}-$ $\left.F\right|^{1 / m}$ converges pointwise to 0 outside a set of Lebesgue measure 0 .

For the proof we need the following elementary fact.

Lemma 3.5. Let $\left\{\alpha_{m}\right\}_{m \geq 1}$ be a positive sequence such that $\alpha_{m} \leq m^{\lambda}$ for some constant $\lambda \in(0, \overline{1})$. Then the function

$$
F(t)=\sum_{m \geq 1} t^{\frac{m}{\alpha_{m}}}
$$

is well-defined and continuous on $[0,1)$.

Proof: By the assumption we have

$$
t^{\frac{m}{\alpha_{m}}} \leq t^{m^{1-\lambda}}, \quad \forall t \in[0,1), m \geq 1 .
$$


By Weierstrass's test, it suffices to check that

$$
\sum_{m \geq 1} \delta^{m^{1-\lambda}}<\infty, \quad \forall \delta \in(0,1) .
$$

Fix $\delta \in(0,1)$ and set $u_{m}=\delta^{m^{1-\lambda}}$. Then

$$
\begin{aligned}
\frac{u_{m}}{u_{m+1}} & =\left(\frac{1}{\delta}\right)^{(m+1)^{1-\lambda}-m^{1-\lambda}} \\
& =\left(\frac{1}{\delta}\right)^{m^{1-\lambda}\left(\left(1+\frac{1}{m}\right)^{1-\lambda}-1\right)} \\
& =\left(\frac{1}{\delta}\right)^{m^{1-\lambda}\left(1+\frac{1-\lambda}{m}+O\left(\frac{1}{m^{2}}\right)-1\right)} \\
& =e^{C m^{-\lambda}+O\left(m^{-1-\lambda}\right)}, \quad\left(C:=\log \left(\frac{1}{\delta}\right)\right) \\
\geq 1+C m^{-\lambda}+O\left(m^{-1-\lambda}\right), & \left(\text { since } e^{t} \geq 1+t, \forall t>0\right) .
\end{aligned}
$$

It follows that

$$
m\left(\frac{u_{m}}{u_{m+1}}-1\right) \geq C m^{1-\lambda}+O\left(m^{-\lambda}\right) \rightarrow \infty, \quad m \rightarrow \infty .
$$

Using Raabe's criterion, we infer that the series $\sum_{m=1}^{\infty} u_{m}$ is convergent. This finishes the proof.

Proof of Theorem 3.4: Fix $z_{0} \in \mathbb{C}^{n}$ and an open ball $U:=\mathbb{B}\left(z_{0}, r\right)$ of $z_{0}$ that satisfies the condition given in (ii). It suffices to prove that for a given $\varepsilon$, there exist an exceptional set $A_{\varepsilon} \subset U$ of measure less than $\varepsilon$ and a measurable function $F_{\varepsilon}$ on $U \backslash A_{\varepsilon}$ such that $\left|r_{m}-F_{\varepsilon}\right|^{1 / m}$ converges to 0 uniformly on $U_{\varepsilon}$. To this end, observe that by (ii), there exist a constant $\lambda \in(0,1)$ and polynomials $q_{m}^{\prime}, q_{m}^{\prime \prime}$ satisfying the following properties:

(i) $r_{m}=p_{m} / q_{m}$, where $q_{m}=q_{m}^{\prime} q_{m}^{\prime \prime}$.

(ii) $\alpha_{m}:=\operatorname{deg} q_{m}^{\prime} \leq m^{\lambda}$ for every $m \geq m_{0}$ and $q_{m}^{\prime \prime}$ is zero free on $U$.

After shrinking $U$ and making a normalization, we may achieve that $q_{m}^{\prime \prime}$ is zero free on a fix neighborhood $V$ of $\bar{U}$ and that

$$
\left\|q_{m}^{\prime}\right\|_{U}=\left\|q_{m}^{\prime \prime}\right\|_{U}=1
$$

It follows that $\left\|q_{m}\right\|_{U} \leq 1$. Then by Bernstein-Markov's inequality we obtain

$$
\sup _{m \geq 1} \frac{1}{m} \log \left\|q_{m}\right\|_{K}<\infty
$$


for every compact subset $K$ of $\mathbb{C}^{n}$. We claim that there exists a compact non-pluripolar subset $X^{\prime}$ of $X$ such that

$$
\sup _{m \geq 1} \frac{1}{m} \log \left\|p_{m}\right\|_{X^{\prime}}<\infty \text {. }
$$

To see this, set

$$
X_{N}=\left\{z \in X:\left|r_{m}(z)-f(z)\right|^{1 / m} \leq N, \forall m \geq 1\right\}, \quad N \geq 1 .
$$

By assumption (i), we have $\bigcup_{N \geq 1} X_{N}=X$. Since $X$ is non-pluripolar, we infer that there must exist $N_{0}$ such that $X_{N_{0}}$ is non-pluripolar. Since $X_{N_{0}}$ is Borel, it must contain a non-pluripolar compact subset $X^{\prime}$. Since $f$ is bounded on $X$, we obtain

$$
\sup _{m \geq 1}\left\|r_{m}\right\|_{X^{\prime}}^{1 / m}<\infty
$$

The claim follows by combining the above inequality with (3.5). Thus, using again Bernstein-Markov's inequality we derive

$$
\sup _{m \geq 1} \frac{1}{m} \log \left\|p_{m}\right\|_{K}<\infty
$$

for every compact subset $K$ of $\mathbb{C}^{n}$. For $m \geq 1$ we define

$$
u_{m}:=\frac{1}{m} \log \left|p_{m+1} q_{m}-p_{m} q_{m+1}\right| .
$$

Using (3.5), (3.6), and simple estimates, we obtain that the sequence $\left\{u_{m}\right\}_{m \geq 1}$ is uniformly bounded from above on compact sets of $\mathbb{C}^{n}$. The key step is to show that $\left\{u_{m}\right\}_{m \geq 1}$ converges uniformly to $-\infty$ on compact sets of $\mathbb{C}^{n}$. To see this, we first observe the trivial equality

$$
u_{m}=\frac{1}{m} \log \left|q_{m+1} q_{m}\right|+\frac{1}{m} \log \left|r_{m+1}-r_{m}\right| .
$$

It follows from (i) and (3.5) that

$$
\lim _{m \rightarrow \infty} u_{m}(x)=-\infty, \quad \forall x \in X .
$$

Since $X$ is non-pluripolar, using the same argument as in the proof of Theorem 3.1, we infer that $\left\{u_{m}\right\}_{m \geq 1}$ must converge uniformly to $-\infty$ on compact sets of $\mathbb{C}^{n}$. In particular, for a given $M>0$, there exists $m(M)$ such that

$$
\sup _{U} u_{m}<-M, \quad \forall m \geq m(M) .
$$

Thus the following estimates hold on $U$

$$
\left|r_{m+1}-r_{m}\right|<\frac{e^{-M m}}{\left|q_{m+1} q_{m}\right|}, \quad \forall m \geq m(M) .
$$


Next, fix $m \geq m_{0}$, we will use an idea from [4] to estimate the size of the subsets of $U$ on which $q_{m+1}^{\prime} q_{m}^{\prime}$ is small. More precisely, for $\delta \in(0,1)$ we set

$$
\begin{aligned}
& X_{m, \delta}:=\left\{z \in U:\left|q_{m+1}^{\prime}(z) q_{m}^{\prime}(z)\right|<\delta^{m}\right\}, \\
& X_{m, \delta}^{\prime}:=\left\{z \in U:\left|q_{m}^{\prime}(z)\right|<\delta^{m / 2}\right\}, \\
& X_{m, \delta}^{\prime \prime}:=\left\{z \in U:\left|q_{m+1}^{\prime}(z)\right|<\delta^{m / 2}\right\} .
\end{aligned}
$$

It is clear that $X_{m, \delta} \subset X_{m, \delta}^{\prime} \cup X_{m, \delta}^{\prime \prime}$. This implies

$$
\lambda_{2 n}\left(X_{m, \delta}\right) \leq \lambda_{2 n}\left(X_{m, \delta}^{\prime}\right)+\lambda_{2 n}\left(X_{m, \delta}^{\prime \prime}\right) .
$$

Recall that $\lambda_{2 n}$ denotes the Lebesgue measure in $\mathbb{C}^{n}$. By Corollary 4.2 in $[\mathbf{1}]$, we have the following estimates

$$
\lambda_{2 n}\left(X_{m, \delta}^{\prime}\right) \leq C_{n, r_{0}} \delta^{\frac{m}{\alpha_{m}}}, \quad \lambda_{2 n}\left(X_{m, \delta}^{\prime \prime}\right) \leq C_{n, r_{0}} \delta^{\frac{m}{\alpha_{m+1}}} .
$$

Here $C_{n, r_{0}}$ is a positive constant depending only on $n, r_{0}$. So for every $m \geq 1$ we get

$$
\lambda_{2 n}\left(X_{m, \delta}\right) \leq C_{n, r_{0}}\left(\delta^{\frac{m}{\alpha_{m}}}+\delta^{\frac{m}{\alpha_{m}+1}}\right)
$$

It follows that

$$
\lambda_{2 n}\left(A_{\varepsilon, r_{0}}\right) \leq C_{n, r_{0}} \sum_{m \geq 1}\left(\delta^{\frac{m}{\alpha_{m}}}+\delta^{\frac{m}{\alpha_{m}+1}}\right),
$$

where $A_{\varepsilon}:=\bigcup_{m>1} X_{m, \delta}$. By assumption (ii) and Lemma 3.5, we can choose $\delta \in(0,1)$ so small such that the right hand side of the last inequality is less than $\varepsilon$.

On the other hand, since $q_{m}^{\prime \prime}$ is zero free on $V$ if $m \geq m_{0}$, the function $u_{m}:=\frac{1}{m} \log \left|q_{m}^{\prime \prime}\right|$ is pluriharmonic on $V$ for every $m \geq m_{0}$. By the normalization (3.4) and Bernstein-Markov's inequality, we obtain that $\sup _{U} u_{m}=0$ for every $m \geq 1$ and that the sequence $\left\{u_{m}\right\}_{m \geq 1}$ is uniformly bounded from above on compact sets of $\mathbb{C}^{n}$. In particular, this sequence does not converges to $-\infty$ uniformly on compact sets of $V$. It follows that this sequence is also uniformly bounded from below on $U$. Thus we get a constant $C^{\prime}>0$ such that

$$
\inf _{U} \frac{1}{m} \log \left|q_{m}^{\prime \prime} q_{m+1}^{\prime \prime}\right|>-C^{\prime}, \quad \forall m \geq m_{0} .
$$

Hence for $z \in U_{\varepsilon}:=U \backslash A_{\varepsilon}$, from (3.7) and (3.8) we deduce

$$
\left|r_{m+1}(z)-r_{m}(z)\right|<\left(\frac{e^{C^{\prime}-M}}{\delta}\right)^{m}, \quad \forall m \geq m(M) .
$$


Hence, for $M$ large enough such that $e^{C^{\prime}-M}<\delta$, by the triangle inequality we see that $\left\{r_{m}\right\}_{m \geq 1}$ is a Cauchy sequence on $U_{\varepsilon}$. Therefore, $r_{m}$ converges uniformly on $U_{\varepsilon}$ to a measurable $F_{\varepsilon}$ such that

$$
\lim _{m \rightarrow \infty}\left\|r_{m}-F_{\varepsilon}\right\|_{U_{\varepsilon}}^{1 / m}=0 \text {. }
$$

We are done.

Remarks. (i) We do not know if the theorem is still true without assumption (ii). On the other hand, by tracking down the above proof, we see that the conclusion of the theorem remains valid if (ii) is omitted whereas (i) is strengthen to

$$
\lim _{m \rightarrow \infty}\left|r_{m}(x)-f(x)\right|^{1 / m^{\gamma}}=0, \quad \forall x \in X,
$$

where $\gamma>1$ is a constant.

(ii) The proof of the theorem also shows that for any open set $D \subset \mathbb{C}^{n}$ on which $r_{m}$ is holomorphic for every $m$, the convergence is rapidly uniformly on compact sets i.e., for every compact subset $K$ of $D$ we have

$$
\lim _{m \rightarrow \infty}\left\|r_{m}-F\right\|_{K}^{1 / m}=0 .
$$

In particular, $F$ is holomorphic on $D$.

(iii) By considering $r_{m}^{\prime}:=1 / r_{m}$, it is easy to see that the theorem still holds if assumption (ii) is written in terms of the degree of the zero sets of $r_{m}$.

The last result of this section may be regarded as a boundary version of Bloom's theorem (Theorem 1.2).

Theorem 3.6. Let $D$ be a bounded domain in $\mathbb{C}^{n}$ and $X \subset \partial D$ be a compact subset. Let $f$ be a bounded holomorphic function on $D$ and $\left\{r_{m}\right\}_{m \geq 1}$ be a sequence of rational functions on $\mathbb{C}^{n}$. Suppose that the following conditions are satisfied:

(i) For every $x \in X$, the point $r x \in D$ for $r<1$ and close enough to 1 . Furthermore, if $u \in \operatorname{PSH}(D), u<0$ and satisfies

$$
\lim _{r \rightarrow 1^{-}} u(r x)=-\infty, \quad \forall x \in X
$$

then $u \equiv-\infty$.

(ii) For every $x \in X$, there exists the limit

$$
f^{*}(x):=\lim _{r \rightarrow 1^{-}} f(r x) .
$$

(iii) The sequence $\left|r_{m}-f^{*}\right|^{1 / m}$ converges pointwise to 0 on $X$. 
Then the following assertions hold true:

(a) The sequence $\left|r_{m}-f\right|^{1 / m}$ converges in capacity to 0 on $D$.

(b) There exists a pluripolar subset $E$ of $\mathbb{C}^{n}$ with the following property: For every $z_{0} \in D \backslash E$ and every affine complex subspace $L$ of $\mathbb{C}^{n}$ passing through $z_{0}$, there exists a subsequence $\left\{r_{m_{j}}\right\}_{j \geq 1}$ such that $\left|r_{m_{j}}-f\right|_{D_{z_{0}}}^{1 / m_{j}}$ converges to 0 in capacity (with respect to $L$ ). Here $D_{z_{0}}$ denotes the connected component of $D \cap L$ that contains $z_{0}$.

Remarks. (i) The first condition imposed on $X$ is local, and if $\partial D$ is $\mathcal{C}^{1}$-smooth at points of $X$ then it is valid after a suitable change of coordinates.

(ii) If $X$ is a compact subset of the unit circle with positive Lebesgue measure then $X$ satisfies assumption (i). This can be seen as follows. Let $u \in \mathrm{SH}(\Delta), u<0$, where $\Delta$ is the unit disk in $\mathbb{C}$ be such that

$$
\lim _{r \rightarrow 1^{-}} u(r x)=-\infty, \quad \forall x \in X .
$$

We have to show that $u \equiv-\infty$. Fix a point $\xi \in \Delta$, by composing with an automorphism of $\Delta$ we may assume that $\xi=0$. Then, using the mean value inequality and Fatou's lemma we obtain

$$
u(0) \leq \frac{1}{2 \pi} \limsup _{r \rightarrow 1^{-}} \int_{0}^{2 \pi} u\left(r e^{i \theta}\right) d \theta \leq \frac{1}{2 \pi} \int_{0}^{2 \pi} \limsup _{r \rightarrow 1^{-}} u\left(r e^{i \theta}\right) d \theta=-\infty .
$$

We are done.

(iii) Part (b) of Theorem 3.6 has not been studied before by either Bloom or Gonchar even in the case where $X$ is a non-pluripolar subset of $D$. It is inspired by a result of Sadullaev in [10] which states that locally a holomorphic function can be rapidly approximated (in measure) if and only if its restriction to every complex line can be rapidly approximated.

(iv) The main difficulty that leads to the passage into subsequence in (b) lies in the fact that the complex subspace $L$ may be disjoint from the non-pluripolar set $X$, thus a direct application of (a) is not possible then.

For the proof of the theorem, we first introduce the following notation: Let $D$ be a bounded domain in $\mathbb{C}^{n}$ and $E$ be a subset of $\partial D$. Then we define the following variant of the relative extremal function

$$
\begin{aligned}
\omega_{R}(z, E, D):=\sup \{\varphi(z) & : \varphi \in \operatorname{PSH}(D), \varphi<0, \\
& \left.\limsup _{r \rightarrow 1^{-}, r x \in D} \varphi(r x) \leq-1, \forall x \in E\right\}, \quad z \in D .
\end{aligned}
$$


Using the above terminology, we have the following lemma which exploits property (i) of the set $X$ given in Theorem 3.6.

Lemma 3.7. Let $D$ be a bounded domain in $\mathbb{C}^{n}$ and $X$ be a subset of $\partial D$. Suppose that $X$ satisfies condition (i) of Theorem 3.6. Then for every sequence $\left\{X_{j}\right\}_{j \geq 1} \subset \partial D$ such that $X_{j} \uparrow X$ we have

$$
\lim _{j \rightarrow \infty} \omega_{R}\left(z, X_{j}, D\right)<0, \quad \forall z \in D .
$$

Proof: Assume that the conclusion is false. Since $\left\{\omega_{R}\left(z, X_{j}, D\right)\right\}_{j \geq 1}$ is a decreasing sequence of non-positive functions, we infer that there exists $z_{0} \in D$ such that

$$
\omega_{R}\left(z_{0}, X_{j}, D\right)=0, \quad \forall j \geq 1 .
$$

Fix $j \geq 1$, for every $k \geq 1$, we can find $\varphi_{k, j} \in \operatorname{PSH}(D), \varphi_{k, j}<0$ such that

$$
\lim _{r \rightarrow 1^{-}} \varphi_{k, j}(r x) \leq-1, \quad \forall x \in X_{j} \text { whereas } \varphi_{k, j}\left(z_{0}\right)>-\frac{1}{2^{k}} .
$$

Now we let $\varphi_{j}:=\sum_{k \geq 1} \varphi_{k, j}$. It is easy to check that

$$
\begin{aligned}
\varphi_{j} \in \operatorname{PSH}(D), \varphi_{j}<0, \lim _{r \rightarrow 1^{-}} \varphi_{j}(r x) & =-\infty, \\
& \forall x \in X_{j} \text { whereas } \varphi_{j}\left(z_{0}\right)>-1 .
\end{aligned}
$$

Next, we set

$$
\varphi(z):=\sum_{j \geq 1} \frac{1}{2^{j}} \varphi_{j}(z), \quad \forall z \in D .
$$

It is clear that $\varphi \in \operatorname{PSH}(D), \varphi<0, \varphi\left(z_{0}\right)>-1$. Now, for a given $x \in X$ we choose $j_{x}$ such that $x \in X_{j_{x}}$. Then we have

$$
\lim _{r \rightarrow 1^{-}} \varphi(r x) \leq \frac{1}{2^{j_{x}}} \lim _{r \rightarrow 1^{-}} \varphi_{j_{x}}(r x)=-\infty .
$$

This is a contradiction and the proof is thereby completed.

We also require some standard facts about compactness in the cone of plurisubharmonic functions.

Lemma 3.8. Let $\left\{u_{m}\right\}_{m \geq 1}$ be a sequence of plurisubharmonic functions defined on a domain $D$ in $\mathbb{C}^{n}$. Suppose that the sequence is uniformly bounded from above on compact subsets of $D$ and does not converge to $-\infty$ uniformly on some compact subset of $D$. Then the following assertions hold:

(a) There exists a subsequence $\left\{u_{m_{j}}\right\}_{j \geq 1}$ converging in $L_{\text {loc }}^{1}(D)$ to a function $u \in \operatorname{PSH}(D), u \neq \equiv-\infty$. 
(b) $\lim \sup _{j \rightarrow \infty} u_{m_{j}} \leq u$ on $D$.

(c) $\lim \sup _{j \rightarrow \infty} u_{m_{j}}=u$ outside a pluripolar subset of $D$.

(d) The set $\left\{z \in D: \lim _{j \rightarrow \infty} u_{m_{j}}(z)=-\infty\right\}$ is pluripolar.

Proof: Assertions (a) and (b) follow from Theorem 3.2.12 in [8]. Next, we apply again Theorem 3.2.12 in $[8]$ to obtain $u=\left(\lim \sup _{j \rightarrow \infty} u_{m_{j}}\right)^{*}$ everywhere on $D$. Hence, by Lemma 2.3 we get (c). Finally, (d) follows easily from (c), so we conclude the lemma.

The final ingredient is a sufficient condition for a sequence of measurable functions converging in capacity to 0 .

Lemma 3.9. Let $\left\{u_{m}\right\}_{m \geq 1}$ be a sequence of plurisubharmonic functions and $\left\{v_{m}\right\}_{m \geq 1}$ be a sequence of measurable functions defined on a bounded domain $D \subset \mathbb{C}^{n}$. Assume that the following conditions are satisfied:

(i) $\left\{u_{m}\right\}_{m \geq 1}$ is uniformly bounded from above.

(ii) There exists a compact subset $X$ of $D$ such that

$$
\inf _{m \geq 1} \sup _{z \in X} u_{m}(z)>-\infty .
$$

(iii) $u_{m}+v_{m}$ converges to $-\infty$ uniformly on compact subsets of $D$. Then the sequence $\left\{e^{v_{m}}\right\}_{m \geq 1}$ converges to 0 in capacity.

Proof: Assume otherwise, then there exists a compact subset $K$ of $D$, a subsequence $\left\{m_{j}\right\}$ and constants $0<\varepsilon<1, \delta>0$ such that

$$
\operatorname{cap}\left(K_{j}, D\right)>\delta, \quad \forall j \geq 1,
$$

where $K_{j}:=\left\{z \in K: v_{m_{j}}<\log \varepsilon\right\}$. It follows from (ii) that $u_{m_{j}}$ does not go to $-\infty$ uniformly on $X$. So using Lemma 3.7 and assumption (i), we may assume, after passing to a subsequence that $u_{m_{j}}$ converges in $L_{\text {loc }}^{1}(D)$ to $u \in \operatorname{PSH}(D), u \not \equiv-\infty$. Next, from assumption (iii) we infer that for every $M$ such that $M+\log \varepsilon>0$ there exists $j_{M} \geq 1$ such that

$$
u_{m_{j}}+v_{m_{j}}<-M, \quad \forall j \geq j_{M}, \forall z \in K .
$$

So for $j \geq j_{M}$ we have the following inclusion

$$
K_{j} \subset L_{j}:=\left\{z \in K: u_{m_{j}}<-M-\log \varepsilon\right\} .
$$

This implies

$$
\operatorname{cap}\left(L_{j}, D\right) \geq \operatorname{cap}\left(K_{j}, D\right)>\delta, \quad \forall j \geq j_{M} .
$$

Let $\omega$ be a small neighborhood of $K$ which is relatively compact in $D$. Then we have

$$
\sup _{j \geq 1}\left\|u_{m_{j}}\right\|_{L^{1}(\omega)}<\infty
$$


According to Definition 2.1, we may choose $\varphi_{j} \in \operatorname{PSH}(D),-1<\varphi_{j}<0$ such that

$$
\int_{L_{j}}\left(d d^{c} \varphi_{j}\right)^{n}>\delta
$$

By a version of Chern-Levine-Nirenberg's inequality (see Theorem 2.1.7 in [2]) we obtain for every $j \geq j_{M}$ the following estimates

$\delta<\int_{L_{j}}\left(d d^{c} \varphi_{j}\right)^{n} \leq \frac{1}{M+\log \varepsilon} \int_{K}\left|u_{m_{j}}\right|\left(d d^{c} \varphi_{j}\right)^{n} \leq \frac{C_{K, \omega}}{M+\log \varepsilon}\left\|u_{m_{j}}\right\|_{L^{1}(\omega)}$.

Here $C_{K, \omega}$ is a positive constant dependent only on $K, \omega$. By letting $M \rightarrow \infty$ and applying (3.9) we get a contradiction. The proof is complete.

Proof of Theorem 3.6: (a) After removing from $X$ a pluripolar subset (possibly empty), we may assume that $r_{m}(z) \in \mathbb{C}$ for every $z \in X$ and $m \geq 1$. Since $f^{*}$ is bounded on $X$, from assumptions (ii) and (iii) we infer that

$$
\sup _{m \geq 1} \frac{1}{m} \log \left|r_{m}(x)\right|<\infty, \quad \forall x \in X .
$$

For $N \geq 1$ we let

$$
X_{N}:=\left\{z \in X: \sup _{m \geq 1} \frac{1}{m} \log \left|r_{m}(z)\right| \leq N\right\} .
$$

It follows that $X=\bigcup_{N>1} X_{N}$. Since $X$ is non-pluripolar, we deduce that there exists $N_{0} \geq 1$ such that $X^{\prime}:=X_{N_{0}}$ is non-pluripolar. Now we write $r_{m}=p_{m} / q_{m}$ with $q_{m}$ is normalized so that $\left\|q_{m}\right\|_{X^{\prime}}=1$. For $m \geq 1$, we define the following plurisubharmonic functions on $D$

$$
u_{m}:=\frac{1}{m} \log \left|p_{m}-q_{m} f\right|, \quad v_{m}:=\frac{1}{m} \log \left|q_{m}\right| .
$$

We claim that the sequence $\left\{u_{m}\right\}_{m \geq 1}$ converges to $-\infty$ uniformly on compact sets of $D$. For this purpose, observe that, since $X^{\prime}$ is nonpluripolar, Bernstein-Walsh's inequality (2.1) implies that the sequence $\left\{v_{m}\right\}_{m \geq 1}$ is uniformly bounded from above on compact sets of $\mathbb{C}^{n}$. By the choice of $X^{\prime}$, using Bernstein-Walsh's inequality (2.1) again, we deduce that the sequence $\frac{1}{m} \log \left|p_{m}\right|$ is uniformly bounded from above on compact sets of $\mathbb{C}^{n}$ as well. It follows, using easy estimates and the assumption on boundedness of $f$, that the sequence $\left\{u_{m}\right\}_{m \geq 1}$ is uniformly bounded from above on compact sets of $D$. For $k, j \geq 1$ we let

$$
X_{k, j}=\left\{x \in X:\left|r_{m}(x)-f^{*}(x)\right|^{1 / m}<1 / j, \forall m \geq k\right\}
$$


By the assumption we have $X_{k, j} \uparrow X$ as $j \rightarrow \infty$. Now, fix a point $z_{0} \in D$. In view of Lemma 3.7 and assumption (i), we can find $k_{j}\left(z_{0}\right)$ (depending on $j$ and $z_{0}$ ) such that

$$
\omega_{R}\left(z_{0}, X_{k, j}, D\right)<0, \quad \forall k \geq k_{j}\left(z_{0}\right) .
$$

On the other hand, for $x \in X_{k, j}$ and $m \geq k$ we have

$$
\limsup _{r \rightarrow 1^{-}} u_{m}(r x)=v_{m}(x)+\frac{1}{m} \log \left|r_{m}(x)-f^{*}(x)\right| \leq A_{1}-\log j .
$$

Here $A_{1}:=\sup _{m \geq 1} \sup _{x \in D} v_{m}(x)$ is a finite constant. Now we set

$$
A_{2}:=\sup _{m \geq 1} \sup _{x \in D} u_{m}(x) .
$$

Then by the above reasoning $A_{2}$ is also a finite constant. By combining all of this and taking into account the definition of $\omega_{R}(\cdot, E, X)$, we arrive at the following estimate

$$
u_{m}\left(z_{0}\right) \leq A_{2}+\left(A_{1}-\log j\right) \omega_{R}\left(z_{0}, X_{k, j}, D\right), \quad \forall m \geq k .
$$

By putting (3.10) and (3.11) together and letting $m \rightarrow \infty$ and then $j \rightarrow \infty$ we derive that

$$
\lim _{m \rightarrow \infty} u_{m}\left(z_{0}\right)=-\infty
$$

Since this is true for every $z_{0} \in D$ and since $\left\{u_{m}\right\}_{m \geq 1}$ is uniformly bounded from above on $D$, by Hartogs' lemma we infer that $u_{m}$ must converge uniformly to $-\infty$ on compact sets of $D$. This is our claim.

Finally, for every $z$ lying outside the pole sets of $r_{m}$ we note the following trivial equality

$$
u_{m}(z):=v_{m}(z)+\frac{1}{m} \log \left|r_{m}(z)-f(z)\right| .
$$

Hence, using Lemma 3.8, we conclude that $\left|r_{m}-f\right|^{1 / m}$ converges to 0 in capacity on $D$.

(b) By what we have proved in (a), the sequence $v_{m}:=\frac{1}{m} \log \left|q_{m}\right|$ is uniformly bounded from above on compact sets of $\mathbb{C}^{n}$. Moreover, $v_{m}$ does not tend to $-\infty$ uniformly on a compact set of $\mathbb{C}^{n}$. Thus, by Lemma 3.8(d), there exists a pluripolar subset $E$ of $\mathbb{C}^{n}$ such that

$$
\lim \sup _{j \rightarrow \infty} v_{m}>-\infty \text { on } D \backslash E .
$$

We will show that $E$ has the desired property. For this, fix a point $z_{0} \in D \backslash E$. Let $L$ be an affine complex subspace passing through $z_{0}$ and 
let $D_{z_{0}}$ be the connected component of $D \cap L$ that contains $z_{0}$. Choose a subsequence $\left\{v_{m_{j}}\right\}_{j \geq 1}$ such that

$$
\inf _{j \geq 1} v_{m_{j}}\left(z_{0}\right)>-\infty .
$$

Let $v_{j}^{\prime}$ and $v_{j}^{\prime \prime}$ be the restrictions to $D_{z_{0}}$ of the two sequences $v_{m_{j}}$ and $\frac{1}{m_{j}} \log \left|r_{m_{j}}-f\right|$. According to the results provided in (a), we have $v_{j}^{\prime}+v_{j}^{\prime \prime}$ converges uniformly to $-\infty$ on compact sets of $D_{z_{0}}$. So using again Lemma 3.8 we conclude that $e^{v_{j}^{\prime}}$ converges rapidly in capacity to 0 in $D_{z_{0}}$. We are done.

\section{Explicit constructions of rapid convergence}

The goal of this section is to provide an example of a sequence of rational functions satisfying the assumptions of Theorem 3.6. More precisely, we will construct a sequence of rational functions $\left\{r_{m}\right\}_{m \geq 1}$ with poles lying outside $\bar{\Delta}$ such that $\left\{r_{m}\right\}_{m \geq 1}$ converges rapidly pointwise to $f^{*}$ on some compact subset of $\partial D$. Here $f^{*}$ is the radial boundary values of a bounded holomorphic function $f$ defined on the unit disk $\Delta$. We begin with a general criterion which guarantees rapid convergence of certain infinite products.

Proposition 4.1. Let $\left\{r_{m}\right\}_{m \geq 1}$ be a sequence of rational functions, $D$ a domain in $\mathbb{C}^{n}$, and $\left\{\beta_{m}\right\}_{m \geq 1}$ a sequence of positive numbers. Suppose that the following conditions are satisfied:

(i) $\left\{r_{m}\right\}_{m \geq 1}$ is locally uniformly bounded on $D$.

(ii) $\lim _{m \rightarrow \infty}\left(\sum_{j=m+1}^{\infty} \beta_{j}\right)^{\frac{1}{m}}=0$.

(iii) There exists a non-pluripolar subset $X$ of $D$ such that for every $x \in X$, there exists a constant $M_{x}>0$ such that

$$
\left|\frac{r_{m}(x)}{r_{m-1}(x)}-1\right| \leq M_{x} \beta_{m}, \quad \forall m \geq 2 .
$$

Then the sequence $\left\{r_{m}\right\}_{m \geq 1}$ converges rapidly uniformly on every compact subset of $D$ to a holomorphic function $f$ on $D$.

Proof: It follows from assumptions (i) and (iii) that the infinite product $r_{1} \prod_{m \geq 1} \frac{r_{m+1}}{r_{m}}$ converges pointwise to a complex valued, measurable function $g$ on $X$. We claim that $r_{m}$ converges rapidly pointwise to $g$ on $X$. For this, we write $f_{j}:=r_{j} / r_{j-1}$ for $j \geq 2$. Fix $x \in X$.

$$
\left|g(x)-r_{m}(x)\right|=\left|r_{m}(x)\right|\left|\prod_{j=m+1}^{\infty} f_{j}(x)-1\right| \text {. }
$$


In view of (iii) we obtain the following estimates when $m$ is sufficiently enough

$$
\begin{aligned}
\left|\prod_{j=m+1}^{\infty} f_{j}(x)-1\right| & =\left|\prod_{j=m+1}^{\infty}\left(1+\left(f_{j}(x)-1\right)\right)-1\right| \\
& \leq \prod_{j=m+1}^{\infty}\left(\left|f_{j}(x)-1\right|+1\right)-1 \\
& \leq \prod_{j=m+1}^{\infty}\left(M_{x} \beta_{j}+1\right)-1 \leq \exp \left(\sum_{j=m+1}^{\infty} M_{x} \beta_{j}\right)-1 \\
& \leq 2 M_{x} \sum_{j=m+1}^{\infty} \beta_{j} .
\end{aligned}
$$

Here we use the inequality $e^{t} \leq 1+2 t, 0 \leq t \ll 1$ and $\sum_{j=m+1}^{\infty} \beta_{j} \rightarrow 0$ as $m \rightarrow \infty$. Notice that, from (i) we get a constant $C_{x}>0$ such that $\left|r_{m}(x)\right| \leq C_{x}$ for every $m \geq 1$. Therefore

$$
\left|g(x)-r_{m}(x)\right| \leq 2 C_{x} M_{x} \sum_{j=m+1}^{\infty} \beta_{j} .
$$

The claim now follows from (ii). Finally, it suffices to apply remark (ii) after the proof of Theorem 3.4 to reach the desired conclusion.

For a precise construction, we first fix a compact subset $F$ of the unit circle $\partial \Delta=\{z \in \mathbb{C}:|z|=1\}$ which is of positive length but is nowhere dense in $\partial \Delta$. In particular, $F$ satisfies condition (i) of Theorem 3.6. This can be done by taking a Cantor set $\mathcal{C} \subset(-1,1)$ with the same property and then taking $F:=\partial \Delta \cap \pi^{-1}(\mathcal{C})$, where $\pi$ is the orthogonal projection from $\partial \Delta$ onto the real axis.

Proposition 4.2. There exist a countable subset $A$ of $\mathbb{C} \backslash \bar{\Delta}$ with $F \subset$ $\bar{A}$, a sequence $\left\{r_{m}\right\}_{m \geq 1}$ of rational functions on $\mathbb{C}$, and a holomorphic function $f: \mathbb{C} \backslash \bar{A} \rightarrow \mathbb{C}$ which is bounded on $\Delta$ such that the following properties holds true:

(a) The poles of $\left\{r_{m}\right\}_{m \geq 1}$ are included in A for every $m \geq 1$.

(b) $\left\{r_{m}\right\}_{m \geq 1}$ converges rapidly uniformly on compact sets of $\mathbb{C} \backslash \bar{A}$ to $f$.

(c) $\left\{r_{m}\right\}_{m \geq 1}$ converges rapidly pointwise on $F=\bar{A} \backslash A$ to $f^{*}$, the radial boundary values of $f$.

(d) $f$ does not extend through any point of $F$ to a holomorphic function. 
The proof proceeds through two lemmas. For the first one, we need to introduce some notation. We index $\left(\mathbb{N}^{*}\right)^{2}=\{(m, n): m, n \geq 1\}$ by the graded lexicographic order, that is $(m, n) \prec(p, q)$ if and only if $m+n<p+q$, or $m+n=p+q$ but $m<p$. Let $\operatorname{ind}(m, n)$ is the index of $(m, n)$ in the ordered sequence. Simple computation gives

$$
\operatorname{ind}(m, n)=\frac{(m+n-2)(m+n-1)}{2}+m .
$$

Hence, $\max (m, n) \geq \sqrt{\frac{\operatorname{ind}(m, n)}{2}}$ for all $m, n \geq 1$.

Lemma 4.3. There exists a double-indexed sequence $\left\{r_{m n}\right\} \subset(0,1)$ such that the corresponding graded lexicographic sequence $\left\{s_{j}\right\}_{j \geq 1}$ satisfies the condition

$$
\lim _{n \rightarrow \infty}\left(\sum_{j=n}^{\infty}\left(1-s_{j}\right)\right)^{\frac{1}{n}}=0
$$

Proof: Fix a real number $a>1$. We will prove that the sequence defined by $r_{j k}=1-a^{-j^{4}-k^{4}}$ satisfies the condition. Indeed, we know that $r_{j k}=s_{\text {ind }(j, k)}$ and $\max (j, k) \geq \sqrt{\frac{\operatorname{ind}(j, k)}{2}}$. We have

$$
\sum_{j=n}^{\infty}\left(1-s_{j}\right)=\sum_{\operatorname{ind}(j, k) \geq n}\left(1-r_{j k}\right) \leq \sum_{j \geq\left\lfloor\sqrt{\frac{n}{2}}\right\rfloor}\left(1-r_{j k}\right)+\sum_{k \geq\left\lfloor\sqrt{\frac{n}{2}}\right\rfloor}\left(1-r_{j k}\right) .
$$

Next, we estimate each term in the last expression. We see that

$$
\begin{aligned}
\sum_{j \geq\left\lfloor\sqrt{\frac{n}{2}}\right\rfloor}\left(1-r_{j k}\right) & =\sum_{j \geq\left\lfloor\sqrt{\frac{n}{2}}\right\rfloor} a^{-j^{4}-k^{4}}=\left(\sum_{j \geq\left\lfloor\sqrt{\frac{n}{2}}\right\rfloor} a^{-j^{4}}\right)\left(\sum_{k \geq 1} a^{-k^{4}}\right) \\
& \leq a^{-\left(\left\lfloor\sqrt{\frac{n}{2}}\right\rfloor\right)^{4}}\left(\sum_{j=0}^{\infty} a^{-j}\right)^{2}=\frac{a^{2}}{(a-1)^{2}} a^{-\left(\left\lfloor\sqrt{\frac{n}{2}}\right\rfloor\right)^{4}} .
\end{aligned}
$$

Hence,

$$
\sum_{j=n}^{\infty}\left(1-s_{j}\right) \leq \frac{2 a^{2}}{(a-1)^{2}} a^{-\left(\left\lfloor\sqrt{\frac{n}{2}}\right\rfloor\right)^{4}} .
$$

The last estimate implies the desired limit.

Lemma 4.4. Let $a=e^{i \theta}$ and $b=r e^{i \xi}$ with $0 \leq \theta, \xi \leq \frac{\pi}{2}$. Then

$$
|1-\bar{b} a| \geq \frac{2}{\pi}|\theta-\xi|
$$


Proof: We write

$$
\begin{aligned}
|1-\bar{b} a| & =\left|1-r e^{i(\theta-\xi)}\right|=\sqrt{(1-r \cos (\theta-\xi))^{2}+(r \sin (\theta-\xi))^{2}} \\
& =\sqrt{1+r^{2}-2 r \cos (\theta-\xi)} \geq|\sin (\theta-\xi)| \geq \frac{2}{\pi}|\theta-\xi|,
\end{aligned}
$$

where in the last estimate we use the inequality $\sin t \geq \frac{2}{\pi} t$ for all $0 \leq$ $t \leq \frac{\pi}{2}$.

Lemma 4.5. There exists a sequence $B=\left\{\alpha_{j}\right\}_{j \geq 1} \subset \Delta$ such that

(a) $F$ is the set of accumulation points of $B$.

(b) $\lim _{n \rightarrow \infty}\left(\sum_{j=n+1}^{\infty}\left(1-\left|\alpha_{j}\right|\right)\right)^{\frac{1}{n}}=0$.

(c) For all $\xi \in F, \sum_{j=1}^{\infty} \frac{1-\left|\alpha_{j}\right|}{\left|1-\bar{\alpha}_{j} \xi\right|}<\infty$. Moreover,

$$
\lim _{k \rightarrow \infty}\left(\sum_{j=k+1}^{\infty} \frac{1-\left|\alpha_{j}\right|}{\left|1-\bar{\alpha}_{j} \xi\right|}\right)^{\frac{1}{k}}=0
$$

Proof: We use an argument of Colwell in [5]. The set $\partial \Delta \backslash F$ is a countable union of disjoint open arcs. Let $A$ be the set of end-points of these arcs. We write

$$
A=\left\{a_{m}, b_{m}: m \geq 1\right\}, \quad\left|a_{m}\right|=\left|b_{m}\right|=1, \quad \varphi_{m}=\arg a_{m}<\arg b_{m}=\psi_{m} .
$$

For each $m, n \geq 1$, we define two double-indexed sequences by

$$
\xi_{m n}=\varphi_{m}+\frac{1}{2}\left(\frac{\psi_{m}-\varphi_{m}}{2 \pi}\right)^{n}, \quad \eta_{m n}=\psi_{m}-\frac{1}{2}\left(\frac{\psi_{m}-\varphi_{m}}{2 \pi}\right)^{n} .
$$

Notice that, for fixed $m, \xi_{m n} \downarrow \varphi_{m}$ and $\eta_{m n} \uparrow \psi_{m}$. We let $t_{m n}:=1-$ $\left(1-r_{m n}\right)\left(\frac{\psi_{m}-\varphi_{m}}{2 \pi}\right)^{n}<1-r_{m n}$ where the $r_{m n}$ are defined in Lemma 4.3. Let us set

$$
c_{m n}=t_{m n} e^{i \xi_{m n}}, \quad d_{m n}=t_{m n} e^{i \psi_{m n}} .
$$

We order two sequences $\left\{c_{m n}\right\}$ and $\left\{d_{m n}\right\}$ by using the graded lexicographic order to get two new sequences $\left\{\tilde{c}_{n}\right\}_{n \geq 1}$ and $\left\{\tilde{d}_{n}\right\}_{n \geq 1}$ respectively. Let $B:=\left\{\alpha_{n}\right\}_{n \geq 1}$ be the following sequence

$$
\tilde{c}_{1}, \tilde{d}_{1}, \tilde{c}_{2}, \tilde{d}_{2}, \ldots, \tilde{c}_{n}, \tilde{d}_{n}, \ldots
$$

It is easily seen that $F$ is the set of accumulation points of $B$ and $\bar{B} \subset\left\{z:|z| \leq 1,0 \leq \arg z \leq \frac{\pi}{2}\right\}$. From Lemma 4.3 we also have 


$$
\begin{aligned}
\lim _{n \rightarrow \infty}\left(\sum_{j=n+1}^{\infty}\left(1-\left|\alpha_{j}\right|\right)\right)^{\frac{1}{n}}=0, \text { since } \\
\sum_{j=n+1}^{\infty}\left(1-\left|\alpha_{j}\right|\right) \leq \sum_{j=\left\lfloor\frac{n}{2}\right\rfloor}^{\infty}\left(1-\left|\tilde{c}_{j}\right|+1-\left|\tilde{d}_{j}\right|\right) \\
=\sum_{\operatorname{ind}(j, k) \geq\left\lfloor\frac{n}{2}\right\rfloor}\left(1-\left|c_{j k}\right|+1-\left|d_{j k}\right|\right) \\
\leq \sum_{\operatorname{ind}(j, k) \geq\left\lfloor\frac{n}{2}\right\rfloor} 2\left(1-r_{j k}\right)=2 \sum_{j \geq\left\lfloor\frac{n}{2}\right\rfloor}\left(1-s_{j}\right) .
\end{aligned}
$$

To prove the last property, we remark that if $\xi=e^{i \theta} \in F$, then $\theta \neq \arg \alpha$ for all $\alpha \in B$. More precisely, $\forall m, n \geq 1$,

$$
\left|\theta-\arg c_{m n}\right| \geq \frac{1}{2}\left(\frac{\psi_{m}-\varphi_{m}}{2 \pi}\right)^{n}, \quad\left|\theta-\arg d_{m n}\right| \geq \frac{1}{2}\left(\frac{\psi_{m}-\varphi_{m}}{2 \pi}\right)^{n} .
$$

On the other hand, $1-\left|c_{m n}\right|=1-\left|d_{m n}\right|=\left(1-r_{m n}\right)\left(\frac{\psi_{m}-\varphi_{m}}{2 \pi}\right)^{n}$. We obtain from Lemma 4.4 the following estimates

$$
\frac{1-\left|c_{m n}\right|}{\left|1-\bar{c}_{m n} \xi\right|} \leq \frac{\pi}{2} \frac{1-\left|c_{m n}\right|}{\left|\theta-\arg c_{m n}\right|} \leq \pi\left(1-r_{m n}\right) .
$$

The same inequality as above holds true for $d_{m n}$. Thus,

$$
\begin{aligned}
\sum_{j=1}^{\infty} \frac{1-\left|\alpha_{j}\right|}{\left|1-\bar{\alpha}_{j} \xi\right|} & =\sum_{m, n=1}^{\infty}\left(\frac{1-\left|c_{m n}\right|}{\left|1-\bar{c}_{m n} \xi\right|}+\frac{1-\left|d_{m n}\right|}{\left|1-\bar{d}_{m n} \xi\right|}\right) \\
& \leq 2 \pi \sum_{m, n=1}^{\infty}\left(1-r_{m n}\right)<\infty .
\end{aligned}
$$

For the last assertion, note that

$$
\begin{aligned}
\sum_{j=k+1}^{\infty} \frac{1-\left|\alpha_{j}\right|}{\left|1-\bar{\alpha}_{j} \xi\right|} & \leq \sum_{\operatorname{ind}(m, n) \geq\left\lfloor\frac{k}{2}\right\rfloor}\left(\frac{1-\left|c_{m n}\right|}{\left|1-\bar{c}_{m n} \xi\right|}+\frac{1-\left|d_{m n}\right|}{\left|1-\bar{d}_{m n} \xi\right|}\right) \\
& \leq 2 \pi \sum_{\operatorname{ind}(m, n) \geq\left\lfloor\frac{k}{2}\right\rfloor}^{\infty}\left(1-r_{m n}\right) .
\end{aligned}
$$

By Lemma 4.3 , the $k$-th root of the last term tends to 0 as $k \rightarrow \infty$. This completes the proof of the lemma.

Proof of Proposition 4.2: Let $B:=\left\{\alpha_{j}\right\}_{j \geq 1} \subset \Delta$ be the sequence in Lemma 4.5. Let us set

$$
f_{j}(z)=\frac{\left|\alpha_{j}\right|}{\alpha_{j}} \frac{\alpha_{j}-z}{1-\bar{\alpha}_{j} z}, \quad r_{m}(z)=\prod_{j=1}^{m} f_{j}(z) .
$$


Let $A:=\left\{\frac{1}{\bar{\alpha}_{j}}: j \geq 1\right\}$. Then the set of accumulation points of $A$ is $F$. We fix a compact set $K \subset \mathbb{C} \backslash \bar{A}$. For $j \geq 1$ and $z \in K$ we have

$$
\left|f_{j}(z)-1\right|=\left|\frac{\left(\alpha_{j}+\left|\alpha_{j}\right| z\right)\left(1-\left|\alpha_{j}\right|\right)}{\alpha_{j}\left(1-\bar{\alpha}_{j} z\right)}\right| \leq M_{K}\left(1-\left|\alpha_{j}\right|\right),
$$

where $M_{K}>0$ depends only on $K$ and $A$. Now, Lemma 4.5(a) and Proposition 4.1 imply the second assertion. For rapid convergence at $\xi \in$ $F$, we write

$$
\left|f_{j}(\xi)-1\right|=\left|\frac{\left(\alpha_{j}+\left|\alpha_{j}\right| \xi\right)\left(1-\left|\alpha_{j}\right|\right)}{\alpha_{j}\left(1-\bar{\alpha}_{j} \xi\right)}\right| \leq 4 \frac{1-\left|\alpha_{j}\right|}{\left|\left(1-\bar{\alpha}_{j} \xi\right)\right|},
$$

when $j$ is big enough. Using the same arguments as in the proof of Proposition 4.1 we obtain

$$
\left|\prod_{j=m+1}^{\infty} \frac{\left|\alpha_{j}\right|}{\alpha_{j}} \frac{\alpha_{j}-\xi}{1-\bar{\alpha}_{j} \xi}-1\right| \leq 8 \sum_{j=m+1}^{\infty} \frac{1-\left|\alpha_{j}\right|}{\left|1-\bar{\alpha}_{j} \xi\right|} .
$$

On the other hand, by Theorem 1 in [5], we have $\lim _{n \rightarrow \infty} r_{n}(\xi)=f(\xi)$. Thus, we can find $M_{\xi}>0$ such that $\left|r_{n}(\xi)\right| \leq M_{\xi}$ for all $n \geq 1$. Hence,

$$
\left|f(\xi)-r_{m}(\xi)\right| \leq 8 M_{\xi} \sum_{j=m+1}^{\infty} \frac{1-\left|\alpha_{j}\right|}{\left|1-\bar{\alpha}_{j} \xi\right|} .
$$

Assertion (c) now follows from Lemma 4.5(c). Finally, we observe that since the zero set of $r_{m}$ is exactly $\left\{\alpha_{1}, \ldots, \alpha_{m}\right\}$ and since every point of $F$ is an accumulation point of $\left\{\alpha_{m}\right\}_{m \geq 1}$, the limiting function $f$ can not be extended holomorphically through any point of $F$. This completes the proof.

\section{References}

[1] S. Benelkourchi, B. Jennane, and A. Zeriahi, Polya's inequalities, global uniform integrability and the size of plurisubharmonic lemniscates, Ark. Mat. 43(1) (2005), 85-112. DOI: 10.1007/ BF02383612.

[2] Z. BLOCKI, The complex Monge-Ampère operator in pluripotential theory, Unfinished lecture notes based on graduate course at Jagiellonian University 1997, last modified: November 2002, available at http://gamma.im.uj.edu.pl/ blocki/publ/ln/wykl.pdf.

[3] T. Bloom, On the convergence in capacity of rational approximants, Constr. Approx. 17(1) (2001), 91-102. DOI: 10.1007/ s003650010011. 
[4] E. M. ČIRKA, Meromorphic continuation, and the rate of rational approximations in $C^{N}$, (Russian), Mat. Sb. (N.S.) 99(141), no. 4 (1976), 615-625.

[5] P. Colwell, On the boundary behavior of Blaschke products in the unit disk, Proc. Amer. Math. Soc. 17 (1966), 582-587. DOI: 10.1090/S0002-9939-1966-0193243-6.

[6] K. R. Davidson, Pointwise limits of analytic functions, Amer. Math. Monthly 90(6) (1983), 391-394. DOI: 10.2307/2975578.

[7] A. A. GonČAR, A local condition for the single-valuedness of analytic functions of several variables, (Russian), Mat. Sb. (N.S.) 93(135) (1974), 296-313, 327.

[8] L. Hörmander, "Notions of convexity", Progress in Mathematics 127, Birkhäuser Boston, Inc., Boston, MA, 1994.

[9] M. Klimek, "Pluripotential theory", London Mathematical Society Monographs. New Series 6, Oxford Science Publications, The Clarendon Press, Oxford University Press, New York, 1991.

[10] A. Sadullaev, A criterion for fast rational approximation in $\mathbf{C}^{n}$, (Russian), Mat. Sb. (N.S.) 125(167), no. 2 (1984), 269-279.

[11] J. Siciak, Extremal plurisubharmonic functions in $\mathbf{C}^{\mathbf{n}}$, Ann. Polon. Math. 39 (1981), 175-211.

Nguyen Quang Dieu and Phung Van Manh:

Hanoi National University of Education

136 Xuan Thuy street

Cau Giay, Hanoi

Vietnam

E-mail address: dieu_vn@yahoo.com

E-mail address: manhlth@gmail.com

Pham Hien Bang:

Thai Nguyen University of Education

Luong Ngoc Quyen, Thai Nguyen

Vietnam

E-mail address: phamhienbang@yahoo.com

Le Thanh Hung:

College of Education

Trung Trac, Vinh Phuc

Vietnam

E-mail address: thanhhungcdsp@gmail.com

Primera versió rebuda el 25 d'agost de 2014, darrera versió rebuda el 29 de gener de 2015 . 